\title{
Vaccine approach for Alzheimer's disease revisited
}

\author{
A follow-up of the patients that took part in a halted Alzheimer's vaccine trial indicates that it might have provided benefit in some cases.
}

\section{Simon Frantz}

New results on the first experimental vaccine for Alzheimer's disease (AD) have provided a glimmer of hope that the strategy could help combat the neurodegenerative disorder.

The concept was dealt a blow when a Phase IIa trial on a vaccine (AN-1792; Elan/ Wyeth) — which targets the amyloid- $\beta(\mathrm{A} \beta)$ peptide that forms the brain plaques in $\mathrm{AD}$ - was halted in January 2002 after four subjects developed inflammation of the central nervous system. In March 2002, Elan withdrew the vaccine from clinical trials after the number of patients with inflammation rose to 18 .

But a follow-up of the trial subjects indicates that the vaccine might have benefited some patients. Data presented at the 9th International Conference on Alzheimer's Disease and Related Disorders in Philadelphia showed a trend in improved memory with vaccine responders compared with placebo. However, other cognitive analyses, such as Mini-Mental State Examination, showed no improvement, but the study was not powered for the various clinical endpoints and was halted after patients received only two doses. Together with four autopsy cases that showed a depletion of brain amyloid plaques, the data indicate that amyloid immunotherapy can elicit an immune response that in some cases could lead to amyloid clearance.

"AN-1792 clearly had a biological response," says Dale Schenk, Chief Scientific Officer at Elan. "Now we need to better understand the observed effects."

Perhaps counterintuitively, some study cohorts found that a positive response correlated with a reduction in brain volume, a factor widely thought to correlate with degeneration in the disease state.

Roger Nitsch from the University of Zurich Medical School, Switzerland, who investigated the Zurich cohort, observed that brain volume was reduced after one year, but that patients responding cognitively had a compensatory increase in brain volume after two years. Nitsch thinks that the loss in volume could be due to the clearance of amyloid plaques accompanied by a concurrent loss in activation of cells called astrocytes that support neuron function. With regards to the recovery in volume, Nitsch says, "in the best of all worlds, this could be a sign of regeneration taking place after amyloid removal.” Nitsch hopes other participating centres will collaborate on similar tests that will confirm or refute this hypothesis.

Ultimate success would not only provide a viable option for controlling the disease, but will also provide positive evidence for the amyloid hypothesis — that is, amyloid plaques are the primary cause of $\mathrm{AD}$ rather than the result of it. "The new studies provide additional proof of concept that $\beta$-amyloid plays a crucial role in Alzheimer's disease," says Nitsch. But Stephen Robinson, Deputy Head, Department of Psychology, Monash University, Australia, takes a different tack. "Many outcomes of the study were either neutral or negative, and collectively they refute the amyloid hypothesis," he says.

A paper by Frank LaFerla and colleagues adds weight to the amyloid hypothesis by showing that treatment with anti-A $\beta$ antibodies in transgenic mice resulted in

\section{Characteristics of COX2 inhibitors questioned}

\section{A health watchdog agency's decision on COX2 inhibitors highlights the difference in opinion about the use of these treatments.}

Sophie Petit-Zeman

The ability of the cyclooxygenase-2 (COX2) inhibitors rofecoxib (Vioxx; Merck) and celecoxib (Celebrex; Pfizer) to treat arthritis with fewer side effects than traditional non-steroidal anti-inflammatory drugs (NSAIDs) has been questioned by the French health products safety agency Agence Française de Sécurité Sanitaire des Produits de Santé (AFSSAPS).

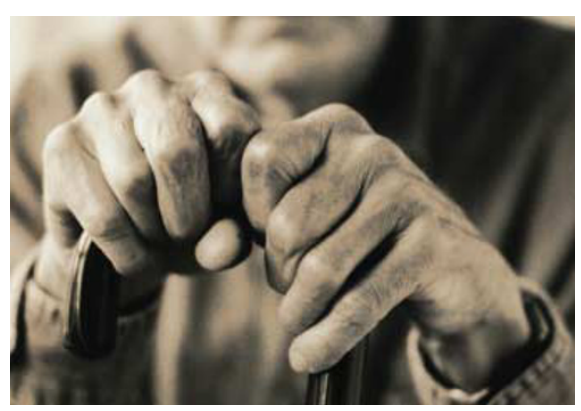

Adverse effects: the use of COX2 inhibitors for arthritis has been questioned.
As Anne Castot, head of AFSSAPS's coordination and management of pharmacovigilance explains, "Concerns about high prescribing levels of COX2 inhibitors, including for elderly patients and those at risk of gastrointestinal (GI) problems, led us to initiate re-evaluation at European Union (EU) level. Considering data from pharmacovigilance and clinical trials worldwide, the benefit/risk ratio remains positive, but prescribing and labelling need caution."

The decision highlights the worldwide debate about the use of COX2 inhibitors. The UK drug watchdog, the National Institute for Clinical Excellence (NICE), recommended in 2001 that all NSAIDs be used cautiously, and COX2 inhibitors preferentially only in patients at high risk of GI side effects. In the United States, COX2 inhibitors are used more widely for pain relief, and US $\$ 6$ billion annually is spent on rofecoxib and celecoxib alone. But fears have recently been raised about reports linking rofecoxib to heart attacks. Some insurers now insist that doctors get 'prior approval' before prescribing such drugs.
This stems in part from a blurring of the divide between the roles of COX1 and COX2. The central idea that inhibition of COX2 is responsible for the therapeutic actions of the drugs, whereas COX1 inhibition is responsible for the side effects, is still valid, although there are some caveats. Reports have indicated that COX2 is more widespread than first suspected and that it is not necessarily restricted to inflammatory sites. For example, the kidney contains high levels of COX2 under 'normal conditions', and so COX2 inhibitors could cause hypertension and fluid retention in some patients.

Jon Deeks, Senior Medical Statistician at the Institute of Health Sciences, Oxford, systematically reviewed COX2 inhibitors (Deeks, J. J. et al. $B M J 325,619$ (2002)), and says there is a general problem in assessing the two large trials VIGOR for rofecoxib and CLASS for celecoxib - which investigated COX2 inhibitors and serious GI adverse events compared with NSAIDs.

"CLASS found fewer adverse events with celecoxib versus NSAIDs during the trial's first 
clearance of $\mathrm{A} \beta$ and a protein called tau (Oddo, S. et al. Neuron 43, 321-332 (2004)). Tau is another potential part of the AD equation, forming neurofibrillary tangles inside brain cells that kill the cells in $\mathrm{AD}$. $\mathrm{A} \beta$ clearance occurred before tau clearance and only early forms of tau were cleared, indicating that early intervention with $A \beta$ immunization might be useful for clearing both hallmark lesions of AD.

But AN-1792 was similarly successful in preclinical studies, only to lead to the Phase IIa results. "The trouble is that mouse models are not $\mathrm{AD}$, and much of this strategy is based on the questionable hypothesis that $\mathrm{AD}$ is caused by $\mathrm{A} \beta$ accumulation," says Patrick McGeer, from the Kinsmen Laboratory of Neurological Research, University of British Columbia, Canada.

In the meantime, Elan and Wyeth are developing 'next-generation' forms of immunotherapy that induce the favourable antibody response but avoid triggering the T-cell response that is thought to lead to the adverse inflammation. Given the lack of unequivocal information, Schenk says that all these issues require pragmatic solutions. "Now that we know that this approach can affect levels of beta-amyloid plaques," he says, "we need to understand what clinical effects this might have for the patients."

six months," says Deeks. But analysis of the data at 12 months is more complicated due to issues such as patient drop-out. Therefore, Deeks believes that the six-month data should be the primary consideration, as other trials strongly indicate that COX2 inhibitors favourably alter outcomes such as endoscopically detectable ulcers. "Australia's decision is most closely linked to the evidence," says Deeks, "approving them for six months based on evidence from CLASS, but with less commitment to longer treatment."

Rod Flower, Professor of Biochemical Pharmacology at St Bartholomew's and the Royal London School of Medicine and Dentistry, says that CLASS, VIGOR and other trials, "strongly suggest that selective COX2 inhibitors produce less side effects."

But he adds that these studies are performed in carefully monitored patient groups and that patients also often self-medicate with over-the-counter NSAIDs to try to achieve a better effect, which eliminates the potential benefits of selective inhibition. "When released into the general population, polypharmacy may result in these drugs having no apparent significant benefit. Perhaps this underlies the French announcement?"

\section{NEWS IN BRIEF}

\section{Lilly updates clinical trials registry}

Eli Lilly has announced that it will update its clinical trials registry to disclose all trial results (see http://www.lillytrials.com). The registry, due to be launched at the beginning the fourth quarter of this year, will include a listing of all active clinical trials for Lilly compounds in Phase III and IV development, and of clinical trials that are for serious and life-threatening conditions. Predefined and secondary outcome measures specified in study protocols will be published, as well as additional safety and efficacy results. Lilly has provided assurance that negative results will also be disclosed in the public registry. The registry will also contain a database of retrospective clinical-trial result summaries for core efficacy and safety registration trials of marketed compounds approved between 1 July 1994 and 1 July 2004.

\section{Sanofi-Aventis merger completed}

On 20 August, Aventis became a controlled subsidiary of Sanofi-Synthélabo, and the merged company was named Sanofi-Aventis. The US Federal Trade Commission approved the takeover bid after the companies agreed to divest some assets and royalty rights. Sanofi will divest the anticoagulant fondaparinux sodium (Arixtra) to GlaxoSmithKline, as well as studies involving the colorectal cancer treatment irinotecan (Campto) that Aventis is carrying out. Sanofi must also divest Aventis's rights to the insomnia drug eszopiclone (Estorra), either to Sepracor or to another agencyapproved buyer. The French Autorité des Marchés Financiers published the definitive results of Sanofi's offers for Aventis (http://www.amf-france.org). The following eight directors of Sanofi-Aventis took office: Jean-Marc Bruel, Jurgen Dormann, Jean-Rene Fourtou, Serge Kampf, Igor Landau, Hubert Markl, Klaus Pohle and Hermann Scholl.

\section{First IND for RNA-interference-based treatment}

Acuity Pharmaceuticals has filed the first Investigational New Drug (IND) application with the US FDA for a treatment based on RNA interference (RNAi). The IND is for Cand5, for treatment of the eye condition wet age-related macular degeneration (wet AMD). The condition is caused by extended growth of blood vessels in the retina; Cand5 silences the production of vascular endothelial growth factor, which is a key factor in the development of wet AMD. Rival firm Alnylam says it plans to begin its own clinical trials in macular degeneration next year.

\section{Universities combine to combat multiple myeloma}

Four academic cancer centres are joining forces to coordinate clinical trials, share resources and pool their findings on multiple myeloma. The Mayo Clinic and the Dana-Farber Cancer Institute in Boston, the H. Lee Moffitt Cancer Center at the University of South Florida and the University Health Network (Princess Margaret Hospital) in Toronto have formed a partnership called the Multiple Myeloma Research Consortium (MMRC). The partnership will share the resources of a centralized tissue and data bank, and MMRC Member Institutions will conduct research in three core areas: genomics, validation and clinical trials. The innovative partnership was founded by Multiple Myeloma Research Foundation President Kathy Giusti and is funded by a grant from the foundation.

\section{Heart drug shows race-specific effect}

A clinical trial of BiDil, developed by NitroMed, was stopped early on 19 July because of its effectiveness when used in addition to normal therapy in African-Americans with heart failure. The drug, a combination of the nitric oxide donor isosorbide dinitrate and the vasodilator hydralazine, is on course to become the first medicine approved for use in a specific ethnic group, with a predicted launch date of 2005. The drug was abandoned after a trial in the 1980 s produced unimpressive results. But, because the data hinted at differences between responses in white and black patients, NitroMed decided to carry out a further clinical trial in more than 1,000 black heart-failure patients in 2001.

\section{Cholesterol combination drug approved}

The US FDA has approved the highly anticipated combination of simvastatin and the cholesteroluptake inhibitor ezetimibe (Vytorin; Merck/Schering Plough). The news, however, has received a mixed reception from cardiologists. Some welcome the convenience of a single pill that can lower low-density lipoprotein (LDL) cholesterol levels without too much worry about the side effects that can result from high doses of some statins. Others are concerned that there are no long-term outcome data available for ezetimibe, and that it is not known whether the LDL cholesterol reduction with Vytorin will result in the same reduction in clinical events as seen with a statin alone.
For more news and analysis go to news@nature.com www.nature.com/news 